\title{
Nuclear Axial Currents in Chiral Effective Field Theory
}

\author{
Alessandro Baroni* \\ Department of Physics Old Dominion University, Norfolk, VA 23529 \\ E-mail: abaro008@odu. edu
}

\begin{abstract}
We discuss the derivation of two-nucleon axial charge and current operators in chiral effective field theory up to one loop. The derivation is based on time-ordered perturbation theory, and accounts for cancellations between the contributions of irreducible diagrams and the contributions due to non-static corrections from energy denominators of reducible diagrams. Ultraviolet divergencies associated with the loop corrections are isolated in dimensional regularization. The resulting axial current is finite and conserved in the chiral limit, while the axial charge requires renormalization. A complete set of contact terms for the axial charge up to the relevant order in the power counting is constructed.
\end{abstract}

The 8th International Workshop on Chiral Dynamics, CD2015 ***

29 June 2015 - 03 July 2015

Pisa,Italy

${ }^{*}$ Speaker. 


\section{Introduction}

Chiral symmetry is an approximate symmetry of Quantum Chromodynamics (QCD), the fundamental theory that describes the interactions of quarks and gluons - the symmetry becomes exact in the limit of vanishing quark masses. Chiral effective field theory $(\chi$ EFT) is the theoretical framework that permits the derivation of nuclear potentials and electroweak currents from the symmetries of QCD - the exact Lorentz, parity, and time-reversal symmetries, and the approximate chiral symmetry. Pions and nucleons (and low-energy excitations of the nucleon, such as the $\Delta$ isobar), rather than quarks and gluons, are the degrees of freedom of $\chi$ EFT. Chiral symmetry requires the pion to couple to these baryons, as well as to other pions, by powers of its momentum $Q$ and, as a consequence, the Lagrangian describing their interactions can be expanded in powers of $Q / \Lambda_{\chi}$, where $\Lambda_{\chi} \sim 1 \mathrm{GeV}$ is the chiral symmetry breaking scale. Classes of Lagrangians emerge, each characterized by a given power of $Q / \Lambda_{\chi}$, or equivalently a given order in the derivatives of the pion field and/or pion mass factors, and each containing a certain number of unknown parameters, the so called low-energy constants (LECs). These LECs could in principle be calculated from the underlying QCD theory of quarks and gluons, but the non-perturbative nature of this theory at low energies makes this task extremely difficult. Hence, in practice, the LECs are fixed by comparison with experimental data.

In the following we focus on nuclear axial charge and current operators. These were originally derived up to one loop in heavy-baryon covariant perturbation theory (HBPT) in a pioneering work by Park et al. [1]. We re-derive [2] them by employing a formulation of time-ordered perturbation theory (TOPT), which accounts for cancellations occurring at a given order in the power counting between the contributions of irreducible diagrams and the contributions due to non-static corrections from energy denominators of reducible diagrams [3]. Because of the different treatment of reducible diagrams in the HBPT and TOPT approaches, we find differences between the operators obtained in these two formalisms. The Hamiltonians describing the interactions of pions, nucleons, and axial field, are derived from the chiral Lagrangians [4], in the canonical formalism and can be written as

$$
\begin{aligned}
H_{I}= & \sum_{n=1}^{3}\left[\left(H_{\pi N N}^{(n)}+H_{2 \pi N N}^{(n)}+H_{3 \pi N N}^{(n)}+\cdots\right)+\left(H_{N N A}^{(n)}+H_{\pi N N A}^{(n)}+H_{2 \pi N N A}^{(n)}+\cdots\right)\right] \\
& +\sum_{m=1}^{2}\left[\left(H_{2 \pi}^{(2 m)}+H_{4 \pi}^{(2 m)}+\cdots\right)+\left(H_{\pi A}^{(2 m)}+H_{3 \pi A}^{(2 m)}+\cdots\right)\right]
\end{aligned}
$$

where the superscript $n$ denotes the power counting $Q^{n}$ and the subscript specifies the number of pion, nucleon, and axial fields entering a given interaction term. As an example we report here 
some of the Hamiltonians

$$
\begin{aligned}
H_{N N A}^{(1)}= & -\frac{g_{A}}{2} \int \mathrm{d} \mathbf{x} \bar{N} \tau_{a} A_{a}^{i} \gamma_{i} \gamma^{5} N, \\
H_{\pi N N A}^{(1)}= & -\frac{1}{4 f_{\pi}} \int \mathrm{d} \mathbf{x} \bar{N} \mathbf{A}_{0} \cdot(\boldsymbol{\tau} \times \boldsymbol{\pi}) \gamma^{0} N, \\
H_{\pi N N A}^{(2)}= & \int \mathrm{d} \mathbf{x} \bar{N}\left[-\frac{1}{2 f_{\pi}}(\boldsymbol{\tau} \times \boldsymbol{\pi}) \cdot \mathbf{A}_{i} \gamma^{i}-\frac{c_{6}}{4 m f_{\pi}}(\boldsymbol{\tau} \times \boldsymbol{\pi}) \cdot \partial_{i} \mathbf{A}_{j} \sigma^{i j}+\frac{2 c_{3}}{f_{\pi}} \mathbf{A}^{i} \cdot \partial_{i} \boldsymbol{\pi}\right. \\
& \left.+\frac{c_{4}}{f_{\pi}}\left(\partial_{i} \boldsymbol{\pi} \times \boldsymbol{\tau}\right) \cdot \mathbf{A}_{j} \sigma^{i j}\right] N, \\
H_{\pi A}^{(2)}= & f_{\pi} \int \mathrm{d} \mathbf{x}\left(\mathbf{A}^{i} \cdot \partial_{i} \boldsymbol{\pi}+\boldsymbol{A}^{0} \cdot \boldsymbol{\Pi}\right),
\end{aligned}
$$

where $g_{A}$ is the nucleon axial coupling constant, $f_{\pi}$ is the pion decay constant, $N$ is the isospin doublet of nucleon fields, $\boldsymbol{\pi}$ and $\boldsymbol{\Pi}$ are, respectively, the isospin triplet of pion fields and their canonical conjugates, and $\mathbf{A}^{\mu}$ is the external axial field. $\boldsymbol{\sigma}$, and $\boldsymbol{\tau}$ are spin and isospin Pauli matrices.

\section{From amplitudes to currents}

The expansion of the transition amplitude for a given process is based on TOPT. Terms in this expansion are conveniently represented by diagrams. We distinguish between reducible diagrams (diagrams which involve at least one pure nucleonic intermediate state) and irreducible diagrams (diagrams which include pionic and nucleonic intermediate states). The former are enhanced with respect to the latter by a factor of $Q$ for each pure nucleonic intermediate state (see below). In the static limit—in the limit $m \rightarrow \infty$, i.e., neglecting nucleon kinetic energies-reducible contributions are divergent. The prescription proposed by Weinberg [5] to treat these is to define the nuclear potential and currents as given by the irreducible contributions only. Reducible contributions, instead, are generated by solving the Lippmann-Schwinger (or Schrödinger) equation iteratively with the nuclear potential (and currents) arising from irreducible amplitudes.

Our formalism is based on this prescription [3]. However, the omission of reducible contributions from the definition of nuclear operators needs to be dealt with care when the irreducible amplitude is evaluated under an approximation. It is usually the case that the irreducible amplitude is evaluated in the static limit approximation. The iterative process will then generate only that part of the reducible amplitude including the approximate static nuclear operators. The reducible part obtained beyond the static limit approximation needs to be incorporated order by order-along with the irreducible amplitude - in the definition of nuclear operators. This scheme in combination with TOPT, which is best suited to separate the reducible content from the irreducible one, has been implemented in Refs. $[6,7,8]$ and is briefly described below. The method leads to nuclear operators which are not uniquely defined due to the non-uniqueness of the transition amplitude offthe-energy shell. While non unique, the resulting operators are nevertheless unitarily equivalent, and therefore the description of physical systems is not affected by this ambiguity [9, 7].

We note that an alternative approach, implemented to face the difficulties posed by the reducible amplitudes, has been introduced by Epelbaum and collaborators [10]. The method, referred to as the unitary transformation method, is based on TOPT and exploits the Okubo (unitary) 
transformation [11] to decouple the Fock space of pions and nucleons into two subspaces, one containing only pure nucleonic states and the other involving states that retain at least one pion. In this decoupled space, the amplitude does not involve enhanced contributions associated with the reducible diagrams. The subspaces are not-uniquely defined, since it is always possible to perform additional unitary transformations onto them, with a consequent change in the formal definition of the resulting nuclear operators. This, of course, does not affect physical representations.

The two TOPT-based methods outlined above lead to formally equivalent operator structures for the nuclear potential and electromagnetic charge and current up to one loop corrections included, which makes it plausible to conjecture that the two methods are closely related.

In what follows, we focus on the method developed in Refs. $[6,7,8]$ and sketch how nuclear operators are derived from transition amplitudes. We are interested in the construction of the twobody weak axial charge and current operators. We will not discuss the aforementioned unitary equivalence between operators corresponding to different off-the-energy-shell extrapolations of the transition amplitudes. This issue has already been addressed in considerable detail in Ref. [7] for the case of the two-body nuclear potential and electromagnetic charge and current operators. Similar considerations apply to the present case.

The starting point is the conventional perturbative expansion for the amplitude

$$
\left\langle f\left|T_{5}\right| i\right\rangle=\left\langle f\left|H_{I} \sum_{n=1}^{\infty}\left(\frac{1}{E_{i}-H_{0}+i \eta} H_{I}\right)^{n-1}\right| i\right\rangle,
$$

where $|i\rangle$ and $|f\rangle$ represent the initial and final states, respectively $\left|N_{1} N_{2} A\right\rangle$ and $\left|N_{1}^{\prime} N_{2}^{\prime}\right\rangle$ (A denotes generically the external axial field), of energies $E_{i}$ and $E_{f}$ with $E_{i}=E_{f}, H_{0}$ is the Hamiltonian describing free pions and nucleons, and $H_{I}$ is the Hamiltonian describing interactions among these particles. The evaluation of this amplitude is carried out in practice by inserting complete sets of $H_{0}$ eigenstates between successive terms of $H_{I}$. Power counting is then used to organize the expansion in powers of $Q / \Lambda_{\chi} \ll 1$.

In the perturbative series, Eq. (2.1), a generic (reducible or irreducible) contribution is characterized by a certain number, say $N$, of vertices, each scaling as $Q^{\alpha_{i}} \times Q^{-\beta_{i} / 2}(i=1, \ldots, N)$, where $\alpha_{i}$ is the power counting implied by the specific term in the interaction Hamiltonian $H_{I}$ under consideration and $\beta_{i}$ is the number of pions in and/or out of the vertex, a corresponding $N-1$ number of energy denominators, and $L$ loops. Out of these $N-1$ energy denominators, $N_{K}$ of them will involve only nucleon kinetic energies and possibly, depending on the particular time ordering under consideration, the energy $\omega_{q}$ associated with the external field, both of which scale as $Q^{2}$, while the remaining $N-N_{K}-1$ energy denominators will involve, in addition, pion energies, which are of order $Q$. Loops, on the other hand, contribute a factor $Q^{3}$ each, since they imply integrations over intermediate three momenta. Hence the power counting associated with such a contribution is

$$
\left(\prod_{i=1}^{N} Q^{\alpha_{i}-\beta_{i} / 2}\right) \times\left[Q^{-\left(N-N_{K}-1\right)} Q^{-2 N_{K}}\right] \times Q^{3 L} .
$$

Each of the $N-N_{K}-1$ energy denominators can be further expanded as

$$
\frac{1}{E_{i}-E_{I}-\omega_{\pi}}=-\frac{1}{\omega_{\pi}}\left[1+\frac{E_{i}-E_{I}}{\omega_{\pi}}+\frac{\left(E_{i}-E_{I}\right)^{2}}{\omega_{\pi}^{2}}+\ldots\right]
$$


where $E_{I}$ denotes the energy of the intermediate state (including the kinetic energies of the two nucleons and, where appropriate, the energy of the external field), and $\omega_{\pi}$ the pion energy (or energies, as the case may be) — the ratio $\left(E_{i}-E_{I}\right) / \omega_{\pi}$ is of order $Q$. The leading order term $-1 / \omega_{\pi}$ represents the static limit, while the sub-leading terms involving powers of $\left(E_{i}-E_{I}\right) / \omega_{\pi}$ represent non-static corrections of increasing order; elsewhere [3,6], we have referred to these as recoil corrections.

Interactions with the external axial field are treated in first order in Eq. (2.1), and inspection of the $Q$ scaling of the various terms shows that the associated transition amplitude admits the following expansion

$$
T_{5}=T_{5}^{(-3)}+T_{5}^{(-2)}+T_{5}^{(-1)}+\ldots
$$

where $T_{5}^{(n)}$ is of order $Q^{n}$. Next, we denote the two-nucleon strong-interaction potential with $v$ and the weak-interaction potential with $v_{5}=A_{a}^{0} \rho_{5, a}-\mathbf{A}_{a} \cdot \mathbf{j}_{5, a}$, where $\rho_{5, a}$ and $\mathbf{j}_{5, a}$ are, respectively, the nuclear weak axial charge and current operators and $A_{a}^{\mu}=\left(A_{a}^{0}, \mathbf{A}_{a}\right)$ is the external axial field. We construct $v+v_{5}$ by requiring that iterations of $v+v_{5}$ in the Lippmann-Schwinger equation [7]

$$
\left(v+v_{5}\right)+\left(v+v_{5}\right) G_{0}\left(v+v_{5}\right)+\left(v+v_{5}\right) G_{0}\left(v+v_{5}\right) G_{0}\left(v+v_{5}\right)+\ldots,
$$

match the $T_{5}$ amplitude, on the energy shell $E_{i}=E_{f}$, order by order in the power counting; here $G_{0}$ denotes the propagator $G_{0}=1 /\left(E_{i}-E_{I}+i \eta\right)$. The potentials $v$ and $v_{5}$ have the following expansions

$$
\begin{aligned}
v & =v^{(0)}+v^{(2)}+v^{(3)}+\ldots \\
v_{5} & =v_{5}^{(-3)}+v_{5}^{(-2)}+v_{5}^{(-1)}+v_{5}^{(0)}+v_{5}^{(1)}+\ldots
\end{aligned}
$$

where the potentials $v^{(n)}$ have been derived in Refs. [6, 7], in particular $v^{(1)}$ vanishes [7], and $v_{5}^{(n)}=A_{a}^{0} \rho_{5, a}^{(n)}-\mathbf{A}_{a} \cdot \mathbf{j}_{5, a}^{(n)}$. Note that hereafter the power counting of $v_{5}^{(n)}$ and $T_{5}^{(n)}$ does not include the factor $Q$ associated with $A_{a}^{\mu}$. The matching between $T_{5}^{(n)}$ and $v_{5}^{(n)}$ leads to the following relations [7]

$$
\begin{aligned}
v_{5}^{(-3)}=T_{5}^{(-3)}, & \\
v_{5}^{(-2)}=T_{5}^{(-2)} & -\left[v_{5}^{(-3)} G_{0} v^{(0)}+v^{(0)} G_{0} v_{5}^{(-3)}\right], \\
v_{5}^{(-1)}=T_{5}^{(-1)} & -\left[v_{5}^{(-3)} G_{0} v^{(0)} G_{0} v^{(0)}+\text { permutations }\right] \\
& -\left[v_{5}^{(-2)} G_{0} v^{(0)}+v^{(0)} G_{0} v_{5}^{(-2)}\right], \\
v_{5}^{(0)}=T_{5}^{(0)} & -\left[v_{5}^{(-3)} G_{0} v^{(0)} G_{0} v^{(0)} G_{0} v^{(0)}+\text { permutations }\right] \\
& -\left[v_{5}^{(-2)} G_{0} v^{(0)} G_{0} v^{(0)}+\text { permutations }\right] \\
& -\left[v_{5}^{(-1)} G_{0} v^{(0)}+v^{(0)} G_{0} v_{5}^{(-1)}\right] \\
& -\left[v_{5}^{(-3)} G_{0} v^{(2)}+v^{(2)} G_{0} v_{5}^{(-3)}\right],
\end{aligned}
$$




$$
\begin{aligned}
v_{5}^{(1)}=T_{5}^{(1)} & -\left[v_{5}^{(-3)} G_{0} v^{(0)} G_{0} v^{(0)} G_{0} v^{(0)} G_{0} v^{(0)}+\text { permutations }\right] \\
& -\left[v_{5}^{(-2)} G_{0} v^{(0)} G_{0} v^{(0)} G_{0} v^{(0)}+\text { permutations }\right] \\
& -\left[v_{5}^{(-1)} G_{0} v^{(0)} G_{0} v^{(0)}+\text { permutations }\right] \\
& -\left[v_{5}^{(0)} G_{0} v^{(0)}+v^{(0)} G_{0} v_{5}^{(0)}\right] \\
& -\left[v_{5}^{(-3)} G_{0} v^{(2)} G_{0} v^{(0)}+\text { permutations }\right] \\
& -\left[v_{5}^{(-2)} G_{0} v^{(2)}+v^{(2)} G_{0} v_{5}^{(-2)}\right] \\
& -\left[v_{5}^{(-3)} G_{0} v^{(3)}+v^{(3)} G_{0} v_{5}^{(-3)}\right]
\end{aligned}
$$

and a similar set of relations is obtained between $T^{(n)}$ and $v^{(n)}$, i.e., the amplitudes and potentials in the presence of strong interactions only [7]. These relations allow us to construct $v^{(n)}$ and $v_{5}^{(n)}$ from $T^{(n)}$ and $T_{5}^{(n)}$.

\section{Tree level and two-pion exchange contributions}

In the following $\mathbf{q}$ is the momentum carried by the external axial field, $\mathbf{k}_{i}$ and $\mathbf{K}_{i}$ denote the combinations of initial and final nucleon momenta $\mathbf{k}_{i}=\mathbf{p}_{i}^{\prime}-\mathbf{p}_{i}, \mathbf{K}_{i}=\left(\mathbf{p}_{i}^{\prime}+\mathbf{p}_{i}\right) / 2$. A symmetrization $1 \rightleftarrows 2$ and an overall momentum-conserving $\delta$-function $(2 \pi)^{3} \delta\left(\mathbf{k}_{1}+\mathbf{k}_{2}-\mathbf{q}\right)$ are understood in the expressions below. We also define $s_{i}=\sqrt{\mathbf{k}_{i}^{2}+4 m_{\pi}^{2}}$.

\subsection{Axial charge}

Tree level and two-pion exchange contributions to the axial charge are illustrated in Fig.1. The lowest order $n=-2$ consists of the single-nucleon axial charge operator

$$
\rho_{5, a}^{(-2)}=-\frac{g_{A}}{2 m} \tau_{1, a} \boldsymbol{\sigma}_{1} \cdot \mathbf{K}_{1} .
$$

The leading order one-pion exchange (OPE) contribution is given by

$$
\rho_{5, a}^{(-1)}=i \frac{g_{A}}{4 f_{\pi}^{2}}\left(\tau_{1} \times \tau_{2}\right)_{a} \boldsymbol{\sigma}_{2} \cdot \mathbf{k}_{2} \frac{1}{\omega_{2}^{2}} .
$$

There are in principle contributions at $Q^{0}$ from $\mathscr{L}_{\pi N}^{(2)}$ and turn out to vanish when summing over all time-orderings. At order $Q^{1}$ we have loop corrections from two-pion exchange (TPE) contributions and are UV divergent. Divergences are isolated in dimensional regularization and reabsorbed in two of the 4 (unknown) LECs $z_{i}$ that enter in the contact axial charge (CT) at this order $\left(Q^{1}\right)$ (a derivation is in [2]).

$$
\rho_{5, a}^{\mathrm{CT}}=\sum_{i=1}^{4} z_{i} O_{i},
$$




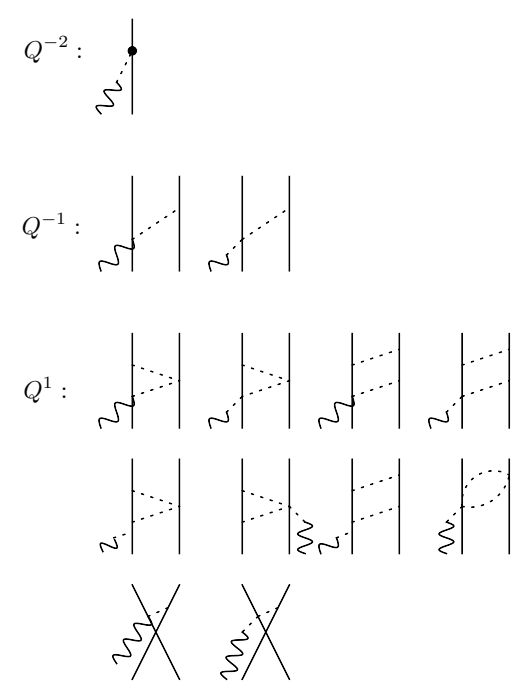

Figure 1: Diagrams contributing to one-body axial charge at leading order $Q^{-2}$, to OPE axial charge at leading order $Q^{-1}$, and to the TPE axial charge operator at order $Q$. Nucleons, pions, and axial fields are denoted by solid, dashed, and wavy lines, respectively. Only a single time ordering is shown for each topology.

where the operators $O_{i}$ have been defined as

$$
\begin{aligned}
& O_{1}=i\left(\boldsymbol{\tau}_{1} \times \boldsymbol{\tau}_{2}\right)_{a}\left(\boldsymbol{\sigma}_{1} \cdot \mathbf{k}_{2}-\boldsymbol{\sigma}_{2} \cdot \mathbf{k}_{1}\right), \\
& O_{2}=i\left(\boldsymbol{\tau}_{1} \times \boldsymbol{\tau}_{2}\right)_{a}\left(\boldsymbol{\sigma}_{1} \cdot \mathbf{k}_{1}-\boldsymbol{\sigma}_{2} \cdot \mathbf{k}_{2}\right), \\
& O_{3}=i\left(\boldsymbol{\sigma}_{1} \times \boldsymbol{\sigma}_{2}\right)_{a}\left(\tau_{1, a} \mathbf{k}_{2}-\tau_{2, a} \mathbf{k}_{1}\right), \\
& O_{4}=\left(\tau_{1, a}-\tau_{2, a}\right)\left(\boldsymbol{\sigma}_{1}-\boldsymbol{\sigma}_{2}\right) \cdot\left(\mathbf{K}_{1}+\mathbf{K}_{2}\right) .
\end{aligned}
$$

\subsection{Axial current}

Non vanishing tree level and two-pion exchange contributions to the axial current are illustrated in Fig. 2. The lowest order $n=-3$ consists of the single nucleon operator

$$
\mathbf{j}_{5, a}^{(-3)}=-\frac{g_{A}}{2} \tau_{1, a}\left[\boldsymbol{\sigma}_{1}-\frac{\mathbf{q}}{q^{2}+m_{\pi}^{2}} \boldsymbol{\sigma}_{1} \cdot \mathbf{q}\right]
$$

where the second term is originated from one-body pion-pole diagrams, and it is necessary for current conservation in the chiral limit. Tree level contributions, due to one-pion exchange diagrams at order $Q^{0}$, read [2]

$$
\begin{aligned}
\mathbf{j}_{5, a}^{(0)}= & \frac{g_{A}}{2 f_{\pi}^{2}}\left(\boldsymbol{\tau}_{1} \times \boldsymbol{\tau}_{2}\right)_{a} \frac{\boldsymbol{\sigma}_{2} \cdot \mathbf{k}_{2}}{\omega_{2}^{2}}\left[\left(c_{4}+\frac{1}{4 m}\right)\left(\boldsymbol{\sigma}_{1} \times \mathbf{k}_{2}-\frac{\mathbf{q}}{q^{2}+m_{\pi}^{2}} \mathbf{q} \cdot\left(\boldsymbol{\sigma}_{1} \times \mathbf{k}_{2}\right)\right)\right. \\
& \left.-\frac{c_{6}+1}{4 m} \boldsymbol{\sigma}_{1} \times \mathbf{q}+\frac{i}{2 m}\left(\mathbf{K}_{1}-\frac{\mathbf{q}}{q^{2}+m_{\pi}^{2}} \mathbf{q} \cdot \mathbf{K}_{1}\right)+\frac{i}{2 m} \frac{\mathbf{q}}{q^{2}+m_{\pi}^{2}}\left(\mathbf{k}_{1} \cdot \mathbf{K}_{1}+\mathbf{k}_{2} \cdot \mathbf{K}_{2}\right)\right] \\
& +\frac{g_{A}}{f_{\pi}^{2}} \tau_{2, a} \frac{\boldsymbol{\sigma}_{2} \cdot \mathbf{k}_{2}}{\omega_{2}^{2}}\left[c_{3}\left(\mathbf{k}_{2}-\frac{\mathbf{q}}{q^{2}+m_{\pi}^{2}} \mathbf{q} \cdot \mathbf{k}_{2}\right)-2 c_{1} m_{\pi}^{2}\right]
\end{aligned}
$$




$$
\begin{aligned}
& Q_{-3}: \text { दो } \\
& Q_{-1}: \text { 2. } \\
& Q_{0}: \text { §... }
\end{aligned}
$$

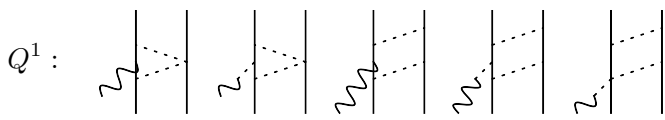

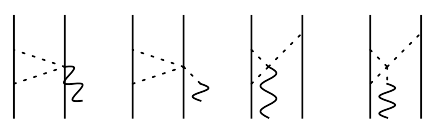

Figure 2: Diagrams contributing to the OPE axial current operator at order $Q^{0}$ and to the multi-pion exchange (MPE) axial current at order $Q$. Nucleons, pions, and axial fields are denoted by solid, dashed, and wavy lines, respectively. Only a single time ordering is shown for each topology. Only non vanishing topologies are reported.

depending on 4 LECs $c_{1}, c_{3}, c_{4}$ and $c_{6}$ coming from $\mathscr{L}_{\pi N}^{(2)}$. It is known that a single contact term occurs at order $Q^{0}$ which we choose as

$$
\mathbf{j}_{5, a}^{\mathrm{CT}}=z_{0}\left(\boldsymbol{\tau}_{1} \times \boldsymbol{\tau}_{2}\right)_{a} \boldsymbol{\sigma}_{1} \times \boldsymbol{\sigma}_{2},
$$

and none at order $Q$. The LEC $z_{0}$ is related to the LEC $c_{D}$ (in standard notation) entering the threenucleon potential at leading order, as first pointed out by the authors of Ref. [12]. The two LECs $c_{D}$ and $c_{E}$ which fully characterize this potential have been recently constrained by reproducing the empirical value of the Gamow-Teller matrix element in tritium $\beta$ decay and the binding energies of the trinucleons $[13,14]$.

\section{Loop corrections to OPE}

Loop corrections to OPE weak axial charge, due to non pion-pole topologies are illustrated in Fig. 3 (only few of them are shown for illustration). Diagrams in classes a and d of Fig. 3 turn out to vanish. Diagrams in classes $b$ and $c$ give a non vanishing contribution, the associated expressions, evaluated in dimensional regularization, are divergent. These divergences have been reabsorbed through a redefinition of LECs involving the $\pi \pi N N$ and $\pi N A$ vertices of order $Q^{3}$ coming from $H_{\pi \pi N N}^{(3)}$ and $H_{\pi N A}^{(3)}$, that gives corrections at order $Q^{1}$ to the LO tree level two-body axial charge. The details of the calculation can be found in Ref. [2]. The final renormalized expression reads 


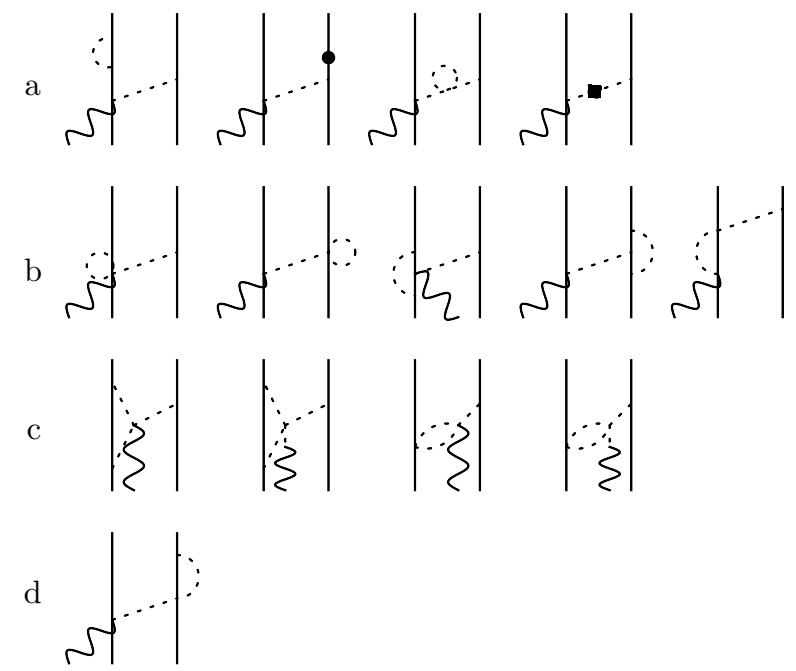

Figure 3: Diagrams contributing to the OPE axial charge operator up to order $Q^{1}$ (a, b , c , d denote the four classes of diagrams that contribute). Nucleons, pions, and axial fields are denoted by solid, dashed, and wavy lines, respectively. Only a single time ordering is shown for each topology.

$$
\begin{aligned}
\rho_{5, a}^{\mathrm{OPE}}= & i \frac{g_{A}^{r}}{4 f_{\pi}^{r 2}}\left(\boldsymbol{\tau}_{1} \times \tau_{2}\right)_{a} \boldsymbol{\sigma}_{2} \cdot \mathbf{k}_{2} \frac{1}{\omega_{2}^{2}}\left[1+\frac{g_{A}^{r 2}}{96 \pi^{2} f_{\pi}^{r 2}}\left[\left(5 k_{1}^{2}+8 m_{\pi}^{r 2}\right) \frac{s_{1}}{k_{1}} \ln \frac{s_{1}+k_{1}}{s_{1}-k_{1}}-\frac{13}{3} k_{1}^{2}+2 m_{\pi}^{2}\right]\right. \\
& \left.+\frac{1}{96 \pi^{2} f_{\pi}^{r 2}}\left(\frac{s_{1}^{3}}{k_{1}} \ln \frac{s_{1}+k_{1}}{s_{1}-k_{1}}-\frac{5}{3} k_{1}^{2}-8 m_{\pi}^{r 2}\right)+\left(\widetilde{d}_{1}^{r} k_{1}^{2}+\widetilde{d}_{2}^{r} k_{2}^{2}+\widetilde{d}_{3}^{r} q^{2}+\widetilde{d}_{4}^{r} m_{\pi}^{r 2}\right)\right] \\
& +i \frac{g_{A}^{r}}{2 f_{\pi}^{r 2}} \widetilde{d}_{5}^{r} \tau_{2, a} \boldsymbol{\sigma}_{1} \cdot\left(\mathbf{q} \times \mathbf{k}_{2}\right) \boldsymbol{\sigma}_{2} \cdot \mathbf{k}_{2} \frac{1}{\omega_{2}^{2}},
\end{aligned}
$$

where $g_{A}^{r}, m_{\pi}^{r}$ and $f_{\pi}^{r}$ denote respectively the physical values of the nucleon axial coupling constant, pion mass and pion decay constant, and their relations with the correspondent bare quantities are given in Eqs. (6.32), (6.8) and (6.18) of Ref. [2]. In particular we note that the relation between $g_{A}^{r}$ and $g_{A}$ obtained in Eq.(6.32) in Ref. [2] matches, to order $Q^{2}$, the one derived in Ref. [15]. The quantities $\widetilde{d}_{i}^{r}$ 's, in Eq. 4.1, denote a linear combination of the physical part of the $d_{i}$ 's entering in $\mathscr{L}_{\pi N}^{(3)}$ Lagrangian, $d_{i}^{r}$.

$$
\begin{aligned}
\widetilde{d}_{1}^{r} & =2 d_{2}^{r}+d_{6}^{r}, \\
\widetilde{d}_{2}^{r} & =4 d_{1}^{r}+2 d_{2}^{r}+4 d_{3}^{r}-d_{6}^{r}, \\
\widetilde{d}_{3}^{r} & =2 d_{6}^{r}-2 d_{2}^{r}, \\
\widetilde{d}_{4}^{r} & =4\left(d_{1}^{r}+d_{2}^{r}+d_{3}^{r}+2 d_{5}^{r}\right), \\
\widetilde{d}_{5}^{r} & =d_{15}^{r}+2 d_{23}^{r} .
\end{aligned}
$$

Some of these LECs have been determined in fits to $\pi N$ scattering data [16]. We observe that the divergent parts of the $d_{i}$ 's, that will be denoted with $d_{i}^{\varepsilon}$, (and hence the $\widetilde{d}_{i}^{\varepsilon}$ 's), have been identified in the heavy-baryon formalism, without considering any specific process, with the backgroundfield and heat-kernel methods, see for example Ref. [17] and references therein. These divergent 
parts match those coming from loop corrections displayed in Fig. 3. In particular $\widetilde{d}_{1}^{\varepsilon}$ cancels the divergent part proportional to $k_{1}^{2}$ coming from the loops (diagrams in class c), $\widetilde{d}_{2}^{\varepsilon}$ and $\widetilde{d}_{4}^{\varepsilon}$ turn out to be identical, therefore can be reabsorbed in the contact term $O_{2}$, and $\widetilde{d}_{3}^{\varepsilon}$ and $\widetilde{d}_{5}^{\varepsilon}$ vanish.

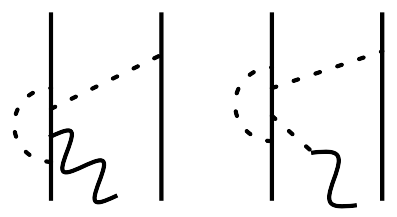

Figure 4: Diagrams contributing to the OPE axial current operator at order $Q^{1}$. Nucleons, pions, and axial fields are denoted by solid, dashed, and wavy lines, respectively. Only a single time ordering is shown for each topology. Only non vanishing topologies are reported.

Loop corrections to OPE weak axial current are illustrated in Fig. 4. The associated integrals are finite in dimensional regularization.

\section{Comparison}

The previous derivation of Ref. [1] has been done in HBPT in the limit of vanishing $\mathbf{q}$, and neglecting the contribution of reducible diagrams in loop corrections, as well some of the irreducible diagrams (i.e. direct box diagram). The present derivation [2], based on TOPT, is done for generic $\mathbf{q}$, and takes into account reducible and irreducible diagrams, present in some of the loop corrections. As a consequence the axial current is conserved in the chiral limit and independent on the parametrization of the pion field. Renormalization of the axial charge operator is accomplished.

\section{Acknowledgments}

A.B. would like to thank the organizers for the workshop invitation, and his collaborators Luca Girlanda, Saori Pastore, Rocco Schiavilla, and Michele Viviani.

\section{References}

[1] T.-S. Park, D.-P. Min, and M. Rho, Phys. Rep. 233, 341 (1993).

[2] A. Baroni, L. Girlanda, S. Pastore, R. Schiavilla, and M. Viviani, Phys. Rev. C 93, 015501 (2016).

[3] S. Pastore, R. Schiavilla, and J.L. Goity, Phys. Rev. C 78, 064002 (2008).

[4] N. Fettes, U.-G. Meissner, M. Mojzis, and S. Steininger, Ann. Phys. (N.Y.) 283, 273 (2000).

[5] S. Weinberg, Phys. Lett. B 251, 288 (1990); Nucl. Phys. B 363, 3 (1991); Phys. Lett. B 295, 114 (1992).

[6] S. Pastore, L. Girlanda, R. Schiavilla, M. Viviani, and R.B. Wiringa, Phys. Rev. C 80, 034004 (2009).

[7] S. Pastore, L. Girlanda, R. Schiavilla, and M. Viviani, Phys. Rev. C 84, 024001 (2011).

[8] M. Piarulli, L. Girlanda, L.E. Marcucci, S. Pastore, R. Schiavilla, and M. Viviani, Phys. Rev. C 87, 014006 (2013).

[9] J.L. Friar, Ann. Phys. (N.Y.) 104, 380 (1977). 
[10] E. Epelbaum, W. Glöckle, and U.-G. Meissner, Nucl. Phys. A 671, 295 (2000); Nucl. Phys. A 714,535 (2003); Nucl. Phys. A 747, 362 (2005).

[11] S. Okubo, Prog. Theor. Phys. 12, 603 (1954).

[12] A. Gardestig and D.R. Phillips, Phys. Rev. Lett. 96, 232301 (2006).

[13] D. Gazit, S. Quaglioni, and P. Navrátil, Phys. Rev. Lett. 103, 102502 (2009)

[14] L.E. Marcucci, A. Kievsky, S. Rosati, R. Schiavilla, and M. Viviani, Phys. Rev. Lett. 108, 052502 (2012).

[15] M. R. Schindler, T. Fuchs, J. Gegelia, and S. Scherer, Phys. Rev. C 75, 025202 (2007).

[16] N. Fettes, U.-G. Meissner, and S. Steininger, Nucl. Phys. A 640, 199 (1998); N. Fettes and U.-G. Meissner, Nucl. Phys. A 693, 693 (2001); H. Krebs, A. Gasparyan, and E. Epelbaum, Phys. Rev. C 85, 054006 (2012); J.M. Alarcon, J.M. Camalich, and J.A. Oller, Ann. Phys. (N.Y.) 336, 413 (2013).

[17] J. Gasser, M.A. Ivanov, E. Lipartia, M. Mojzis, and A. Rusetsky, Eur. Phys. J. C 26,13 (2002). 